\title{
Zur Rolle von Stereotypisierungen bei Assimilations- und Akkommodationsprozessen
}

\author{
Jörg Roche (München)
}

\begin{abstract}
Meaning is widely understood as being a mental construct generated and negociated by means of viabalisation processes. It constantly develops and changes through processes of assimilation and accommodation. How then can stereotypes develop in such a dynamic system of knowledge management where language is a decisive tool in information transfer? The information transfer based on established parameters contributes to a stabilization of meaning and, as a consequence, serves as a point of reference for further thinking, speaking and doing. Linguistic relativity models have shown how this can in turn promote the dynamics of perception/thinking or, equally well, determine or inhibit it. The aim of the present paper is to show how intercultural language teaching can be conceived as a means to foster dynamic perception and mental construction processes of "the other" while leading to the cultural state of 'transdifference' (as opposed to a mere binary contrast of "self" and "other"). Owing to recent and ongoing political events the topic of ,violence and resistance' has been chosen to illustrate the approach.
\end{abstract}

\section{Von der interkulturellen Hermeneutik zur Transkulturation und Transdifferenz}

Das Thema der Tagung kam vor vielen Jahren im Rahmen meiner Tätigkeit als Mittler der deutschen Sprache und Kultur in Kanada zu mir, zu einer Zeit, als die interkulturelle Hermeneutik en vogue war. Zwar ging es mir und meinen Kollegen nicht um modische Strömungen, aber der interkulturelle Kontakt von Sprachen und Kulturen hatte für uns etwas sehr Spannendes, zumal wir damals der festen Überzeugung waren, „das Eigene“ und „das Fremde“ durch einen mehr oder weniger konstanten „Perspektivenwechsel“ zwischen „Innen- und Außenperspektiven“ vereinbaren zu können. Jedenfalls wollten wir unsere Lerner dazu bewegen, über den Fremdsprachenunterricht ihre Stereotypen zu modifizieren oder aufzugeben, in Bezug auf nationale Stereotypen, aber auch in Bezug auf Stereotypisierungen im Umgang mit Identität, Geschichte, Gewalt und anderem. Dass dies gar nicht einfach, oder in der Praxis so gar nicht möglich ist, erfährt man freilich erst, wenn man es im eigenen Fremdkultur- und Fremdsprachenerwerb im eigenen Kopf erlebt. Die teleologische Fusion zu einem „,besseren“ oder „tieferen“ Verständnis des „Fremden und des Eigenen“, wie es seitdem - und bis heute im Sinne der interkulturellen Hermeneutik gerne in Lehrplänen heißt, schien schon damals nicht realistisch, aber es gab keinen besseren Begriff als „,interkulturell“ dafür. Viele Kollegen und auch ich haben ihn immer eher im Sinne einer fluiden Interkulturalität verwendet, weil

Linguistik online 79, 5/2016 - http://dx.doi.org/10.13092/lo.79.3340

CC by 3.0 
uns irgendetwas daran zu statisch und hermetisch schien. Nachdem Welsch $(2010,1999)$ in den 1990er Jahren die Binaritätsannahmen des Eigenen und des Fremden auf Herders Kulturmodell zurückgeführt und als Alternative das Konzept der ,Transkulturalität‘ vorgeschlagen hat, schien man endlich auch einen angemessenen Namen für das gewisse Unwohlsein mit der Inter-Kulturalität gefunden zu haben. Dass dadurch aber das Problem der Statik nur begrifflich, nicht aber auch konzeptuell bewältigt ist, ist zwar in der Fachliteratur inzwischen weitestgehend anerkannt, hat sich aber in der Kulturpraxis noch nicht überall durchgesetzt. Erst der von Ortiz (1995) eingeführte Begriff ,Transkulturation', der die Prozesse des Verstehens bezeichnen will, scheint diese Beschränkungen auf Zustandsbeschreibungen zu überwinden.

Zu den Stereotypen über „Deutsche“ und „Deutschland“, die wir beispielsweise in Nordamerika ermittelt haben, gehörten regelmäßig diverse und diffuse Elemente aus den Begriffsfeldern Autobahn, Bier/Oktoberfest und Nazi-Deutschland. Sauerkraut und Weißwurst sind wohl ältere Klassiker, aber man kann sich sicher auch ohne weitere Beispiele noch einige aktuellere und wichtigere dazu vorstellen. Manche Stereotypen decken sich mit solchen über Deutsche und Deutschland in anderen Ländern - und das zeigen ja auch die Erhebungen aus dem SMIK-Projekt in Dänemark sehr deutlich - und auch mit nationalen Stereotypen über andere Länder. Viele finden sich in der einen oder anderen Weise auch in Autostereotypen der Deutschen wieder und lassen sich oft gar nicht so leicht von faktischen Feststellungen wie etwa dem Bierkonsum oder der Fahrpraxis auf der Autobahn unterscheiden. Oft sagen Stereotype auch mehr aus über die Stereotypisierenden als über die Stereotypisierten aus (vergleiche auch die Gegenüberstellung der Einschätzung von kommunikativen Praktiken von AngloAmerikanern und Athabascans bei Roche 2013; Scollon/Scollon 1990). Alle haben einen unbestimmten Grad an Wahrheit und Unwahrheit, sind also richtig und falsch zur gleichen Zeit. Das weiß man auch landläufig. Stabilisierte Wahrnehmungen betreffen selten eine nationale Gesamtheit, sondern meistens nur regionale und gesellschaftliche Teilgruppen und dies auch nur in bestimmten Zeiträumen. Sie sind also variabel. Das ist nichts Überraschendes.

Bedeutung ist bekanntlich nichts objektiv und fixiert Gegebenes, sondern etwas auf Basis subjektiv abgebildeter Wissens- und Erfahrungsbestände subjektiv Konstruiertes, das mittels Überprüfungs- und Bestätigungsprozessen (Viabilisierungsprozessen) verhandelt wird und sich ständig verändert. Zumindest solange sich ein Individuum irgendwie mit seiner Umwelt auseinandersetzt. Bedeutungskonstruktionen entstehen in diesem eigentlich kontinuierlichen und intuitiv verlaufenden Prozess durch ständige Assimilation neuer Information an bereits vorhandene Wissensbestände und durch Akkommodation bestehender Bedeutungsmuster an neue Information.

Diese Prozesse stellt Ifenthaler (2006) folgendermaßen dar: 


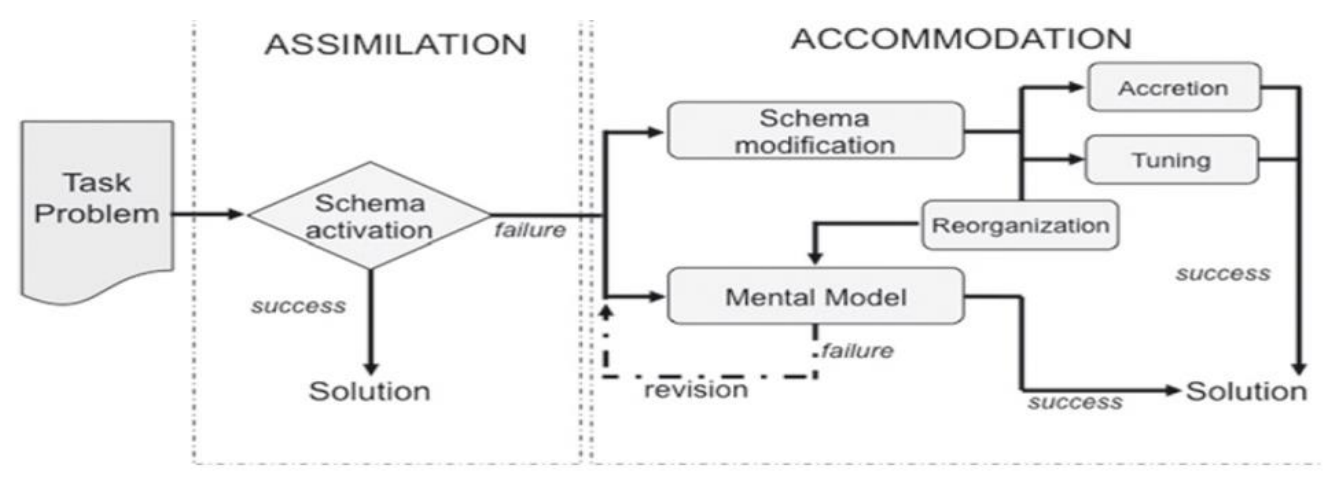

Abbildung 1: Prozessmodell zur Veränderung mentaler Modelle und der Restrukturierung kognitiver Schemata nach Ifenthaler (2006)

Die Dynamik des Wissensmanagements ist in unterschiedlichem Maße durch implizit und explizit tradierte Information - und weil Sprache und Wissen untrennbar zusammen gehören - durch das Transmissions- und Gestaltungsmittel Sprache beeinflusst. Diese Information fördert die Stabilisierung etablierter Bedeutungen, die ihrerseits die weitere Wahrnehmung und das Handeln steuern. Die daraus entstehende (temporäre) Orientierung kann sich positiv auf die weitere Wahrnehmungsdynamik auswirken, oder eine solche auch hemmen oder verhindern. Temporäre Stereotype sind in gewisser Weise der Normalfall der Wahrnehmung. Stabil bleiben können Zwischenprodukte eigentlich nur, wenn es in der automatisierten Dynamik keine hinreichenden Herausforderungsimpulse gibt oder diese kategorisch oder selektiv abgeschottet werden. Sprachliche Stereotypen entwickeln jedoch häufig eine eigene Qualität, die sich verselbständigt. Die ursprüngliche Bedeutung geht zum Teil verloren. So zum Beispiel die Genus/Sexus-Information in ,Studentenwerk', das natürlich für alle Geschlechter und Genera zuständig ist. In Nordrhein-Westfalen ist gerade regierungsamtlich verordnet worden, dass im Hochschulbereich eine geschlechtsneutrale Sprache zu verwenden sei. Dabei werden weder die Geschlechtsneutralität in den Begriffen oben, noch die semantischen Unterschiede zwischen Studierenden und Studentinnen berücksichtigt. Das Studentenwerk in ein Studierendenwerk umzubenennen, verlangt daher mehr als nur Gewöhnungszeit, Studierendenwerksbeitrag und andere Ableitungen noch mehr.

Dass es unterschiedliche Stereotypen gibt, die neben anderen auch über längere Zeiträume im kollektiven Gedächtnis bestehen bleiben können, ist inzwischen eine bekannte Tatsache. Interessant daran ist aber die Frage, wann welche, und warum gerade die stereotypen - trotz konträrer Eigen-Erfahrungen etwa eines Auslandssemesters - aus der kollektiven Wahrnehmung einer fremden Kultur oft bevorzugt aktiviert werden (siehe die Erläuterung des Verhaltens japanischer Austauschstudenten unten).

\section{Zur Sensibilisierung in der Fremdsprachendidaktik}

In der Sprach- und Kulturvermittlung muss man Optimist bleiben und als solcher gehe ich davon aus, dass es neben stabilisierten Wahrnehmungsmustern auch instabile Varianten gibt und im didaktischen Sinne geben sollte. Im Folgenden will ich daher zeigen, wie mit unterschiedlichen, kontrastierten Wahrnehmungsstabilisierungen in einem interkulturell ausgerichteten Unterricht zu einer Dynamisierung der Wahrnehmung („Perspektivenwechsel und - 
vielfalt", ,Transdifferenz") beigetragen werden kann. Aus gegebenem Anlass wird das didaktische Verfahren an Materialien zum Thema ,Gewalt und Widerstand“ illustriert.

Warum dieses Thema? Es ist nur indirekt der traurigen Aktualität zu schulden, aber es zeigt nicht zuletzt in den beklagenswerten Ereignissen der letzten Wochen, wie notwendig es ist, sich damit kontinuierlich auseinanderzusetzen. Als ich mit meinem kanadisch-amerikanischen Kollegen Mark Joel Webber vor vielen Jahren bei Yale University Press ein Text-Buch zu German Studies und damit zu vielen zentralen Aspekten des Umgangs mit Stereotypen vorbereitet habe (Roche/Webber 1995), gab es in der Landeskunde eigentlich nur zwei gängige Modelle für die allseits gewünschte Behandlung und Veränderung von nationalen Stereotypen: 1. die Aufklärung über Faktendarstellungen und 2. die - ich will es mal salopp so nennen, wie es oft praktiziert wurde - die gefühlte Betroffenheits-Relativierung, nach dem „One World Prinzip“ (ausgedrückt unter anderem auch durch Symbolisierungen wie sie in der Benetton-Werbung verwendet wurden oder durch Band Aid - Texte des Bedauerns, dass es dieses Jahr zu Weihnachten in Afrika nicht schneien würde). Die Relativierung stereotyper Wahrnehmungen durch Konkrete Poesie, wie sie etwa der von mir hoch geschätzte Kollege Dietrich Krusche in der damaligen Zeit vor allem in verschiedenen aufsehenerregenden Materialsammlungen bei Inter Nationes vorbereitet hatte (Krusche/Krechel 1984), sind leider nicht in die Hauptströmungen der Lehrwerke aufgenommen worden. Auch die Kontrastierung unterschiedlicher Stereotype mit dem Ziel des Perspektivenwechsels gehörte zum damaligen Zeitpunkt noch nicht zu den gängigen Verfahren: sie war erst im Kommen und in ihrer Statik grundsätzlich nicht allzu anders als das, was man aus der faktenbasierten Landeskunde kannte. „Und wie ist das in Ihrem Land?“ Dabei besteht übrigens auch eine gewisse Gefahr, so wie in der interkulturellen Pädagogik oder im interkulturellen Training, dass durch die zugeschriebene Fremdheit eine Lernumgebung geschaffen wird, in der der Andere fremder oder stereotyper sein muss, als ihm das lieb ist. Zudem gibt es gravierende wissenschaftsmethodische und kulturwissenschaftliche Einwände gegenüber den deterministischen, kultursemantisch und -pragmatisch nicht differenzierenden Ansätzen wie sie von Thomas (2005), Bennett (1993), Trompenaars (1993), Hofstede (1991) oder Hall (1981, 1959) vorgeschlagen wurden.

Wir experimentierten dagegen mit dem, was man als kulturübergreifende Aspekte der Wahrnehmung und als grundlegende Konzepte der menschlichen Existenz bezeichnen könnte, etwa mittels Themen wie Gewalt und Widerstand, Identitäten, Emotionen. Zwar haben wir auch ein umfangreiches, nach wie vor aktuelles Kapitel zu nationalen Stereotypen entwickelt, aber es ging uns immer auch darum, die Lerner dafür zu sensibilisieren, wie sich nationale Stereotypisierungen in wichtigen Bereichen der menschlichen Existenz manifestieren und zum Beispiel auf die Wahrnehmung und Ausgestaltung verschiedener individueller und kollektiver Rollen/Identitäten auswirken. Heute könnte man diese Bereiche im Sinne der Kognitiven Linguistik auch als „Basisdomänen“ bezeichnen und sollte sie so auch behandeln. Hierzu gehören neben den oben genannten auch Domänen wie Leben und Tod, Raum, Zeit und andere. Die Geschichte der Menschheit und ihre sprachlichen Produktionen zeugen von der existenziellen Bedeutung dieser Grundkategorien menschlichen Wahrnehmens, Denkens, Handelns, Sprechens und Schreibens. Ein kleiner, eigentlich banaler didaktischer Vorteil, der andernfalls für Unterrichtspraktiker und Verlage oft zu einem Ablehnungsargument bei der Themenauswahl wird: diese Texte veralten nie und bleiben immer aktuell, selbst wenn die Ereignisse dutzende, hunderte oder tausende von Jahren zurückliegen. Unsere eigentliche Intention je-

ISSN 1615-3014 
Akkommodationsprozessen

doch war, Themen und Texte zu finden, die jeden betreffen und zu denen sich jede und jeder verhalten muss, in seiner/ihrer Erst- oder der Fremdsprache. Und es ging uns nicht um ein „Belehren“ oder „Befüllen“ ignoranter Lernerinnen und Lerner - und damit nicht um ein richtig oder falsch -, sondern um die Vermittlung von kritischer Kompetenz, verstanden als die Fähigkeit, Dinge als relevant zu identifizieren und selbständig und kritisch zu reflektieren, auf Relevanz zu bewerten und zu integrieren. Hier ein einfaches Beispiel, damit deutlich wird, was wir im Unterricht erreichen wollten. Wir haben uns dabei an den Prinzipien der modernen Sprachdidaktik orientiert, die ja versucht, über eine Aufgabenorientierung authentisches sprachliches Handeln im Unterricht abzubilden und für einen fundierten und schnellen Spracherwerb zu instrumentalisieren. Zugegebenermaßen verlangen unsere Aufgaben auch nach anspruchsvollen intellektuellen Lösungen, wie die Beispiele aus Für- und Widersprüche (Roche/Webber 1995) in Abbildung 2, 3 und 4 zeigen:

Haupttext 1: Definitionen von Gewalt, Terror und Widerstand

Dieser Teil des Kapitels stellt einige grundlegende Texte zu den Themen "Gewalt", "Widerstand" und "Terror" vor. Sie finden also einen Text aus der Bibel, Grundgesetzartikel zum Widerstandsrecht, Verfassungsschutzbericht und Wörterbuchdefinitionen.

Ü1 Was assoziieren Sie mit folgenden Begriffen?
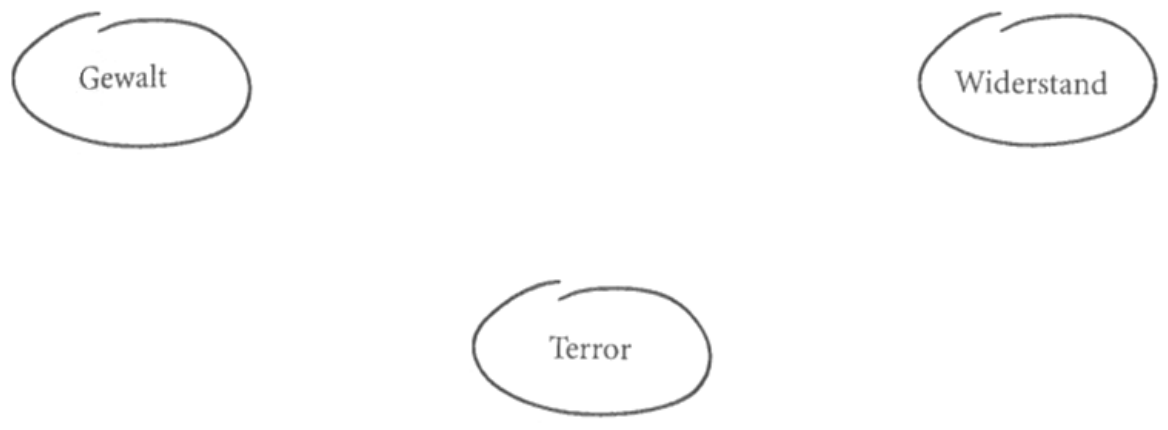

Oberschneiden sich Ihre Assoziationen zu den drei Begriffen? Wie? In welchem Verhältnis stehen die Begriffe zueinander?

Abbildung 2: Aufgabe zu „Gewalt“, „Terror“" und „Widerstand“ (aus Roche/Webber 1995) 
Ü3 Vergleichen Sie die verschiedenen Formulierungen miteinander.

Die Gewalt

Die Gewalt fängt nicht an wenn einer einen erwürgt Sie fängt an wenn einer sagt:

"Ich liebe dich:

Du gehörst mir!"

Die Gewalt fängt nicht an wenn Kranke getötet werden Sie fängt an

wenn einer sagt:

"Du bist krank:

Du mußt tun was ich sage"

Die Gewalt fängt an

wenn Eltern

ihre folgsamen Kinder beherrschen und wenn Päpste und Lehrer und Eltern Selbstbeherrschung verlangen

Die Gewalt herrscht dort

wo der Staat sagt:

“Um die Gewalt zu bekämpfen darf es keine Gewalt mehr geben außer meiner Gewalt"

Die Gewalt herrscht

wo irgendwer

oder irgendetwas

zu hoch ist

oder zu heilig

um noch kritisiert zu werden oder wo die Kritik nichts tun darf sondern nur reden und die Heiligen oder die Hohen mehr tun durfen als reden

Die Gewalt herrscht dort wo es heißt: "Du darfst Gewalt anwenden" aber oft auch dort wo es heißt: "Du darfst keine Gewalt anwenden"

Die Gewalt herrscht dort wo sie ihre Gegner einsperrt und sie verleumdet als Anstifter zur Gewalt

Das Grundgesetz der Gewalt lautet: "Recht ist, was wir tun. Und was die anderen tun das ist Gewalt"

Die Gewalt kann man vielleicht nie mit Gewalt überwinden aber vielleicht auch nicht immer ohne Gewalt

Erich Fried

Abbildung 3: Weitere Aufgabe zu „Gewalt““ (aus Roche/Webber 1995) 
Und hier der vollständige Text:

Warum wehrt ihr euch nicht, rief der Adler den Schafen zu, als er sah, wie der Wolf eins nach dem anderen riß. Schafe, die sich gegen Wölfe wehren, sind gegen die Natur, schrie eins zurück und ließ sich fressen.

Ü3 Hatten Sie mit einer anderen Fortführung beziehungsweise mit einem anderen Schluß gerechnet? Warum? Vergleichen Sie Ihre Vorschläge mit denen Ihrer Kommilitoninnen und Kommilitonen.

Ü4 Identifizieren Sie sich mit einem der Charaktere im Text? Mit welchem? Begründen Sie bitte.

Ü5 Welche Assoziationen verbinden Sie mit Adlern, Wölfen und Schafen?
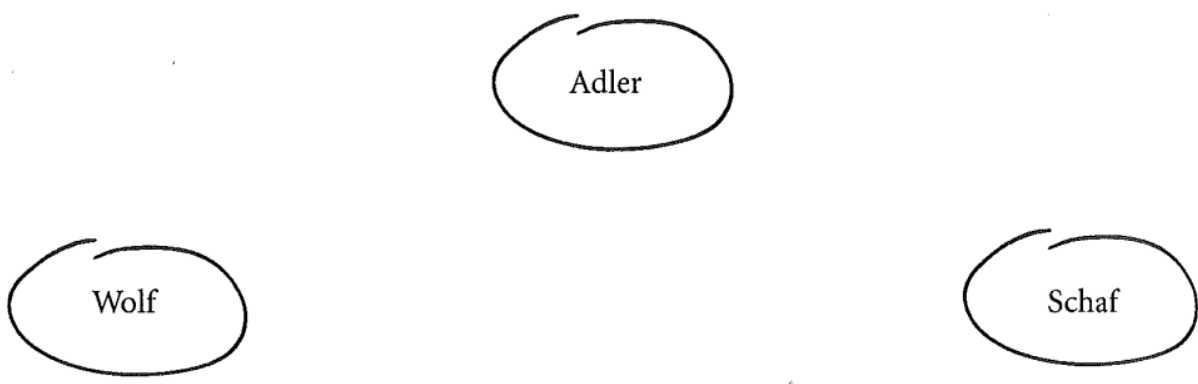

Ü6 Hausaufgabe: Welche Thesen liegen diesem Text zugrunde? Stellen Sie Hypothesen auf?

\section{Abbildung 4: Aufgaben zur Leseerwartung- und Identifikation (aus Roche/Webber 1995)}

Worum ging es also? Folgende Ziele haben die Materialien:

- die Sensibilisierung für Stereotypisierungen, Stabilisierungen und den Mangel an Differenzierung in der Wahrnehmung;

- $\quad$ nicht national-spezifische Themen zu präsentieren, sondern grundlegende Themen, alle historisch relevant, in diesem Fall zu ,Gewalt und Widerstand', in denen sich auch kulturspezifische Stereotypisierungen finden;

- die historische Bedeutung im historischen Kontext erfassen zu lassen;

- das Abholen der Lerner bei ihren vorgefassten Einstellungen, ohne eine Tabula rasa zu erwarten;

- zu Bewertungen, Einordnung/Integration anzuleiten und damit Bedeutung zu konstruieren und zu erweitern;

- zu helfen, persönliche Handlungskonsequenzen zu entwickeln;

- Weiterbeschäftigung mit der Thematik und den Prozessen zu initiieren;

- eine Sensibilisierung für Stabilisierungsprozesse, Kontextabhängigkeit und Differenz/Variation. 


\section{Bedeutungskonstruktion}

Wie entsteht Bedeutung? Durch die Sinnesorgane werden verschiedene Eingangsinformationen, wie Schall- und Lichtwellen, aufgenommen und zur Weiterverarbeitung an die verschiedenen Bereiche des Gehirns weitergeleitet. So wird visuelle Information als Lichtenergie wahrgenommen und über einen photochemischen Prozess in Nervenimpulse umgewandelt. Über die Nervenbahnen gelangt die Information dann zur Sehrinde ins Gehirn. Ähnliche Prozesse laufen bei der Verarbeitung von Schallwellen (auditive Information), Informationen über den Tastsinn (taktile Information), Informationen über den Geruchssinn (olfaktorische Information) und Informationen über den Geschmackssinn (gustatorische Information) ab. Dabei werden bereits Vorentscheidungen getroffen, welche Teile der eingehenden Informationen wichtig sind und welche nicht. Die Tatsache, dass lexikalische Aktivierungen andere Sinneskanäle ko-aktivieren und durch andere Sinneskanäle ko-aktiviert werden können, deutet auf eine mehrfache Kodierung der lexikalischen Information im mentalen Lexikon.

Die eingehende Information wird dazu in den zuständigen Bereichen des Gehirns verarbeitet: Die Informationen, die zusammengehören, kommen dort in unterschiedlicher Form und unterschiedlich schnell an und werden auch unterschiedlich schnell verarbeitet, dennoch erscheinen sie als eine Einheit. Bei der Interpretation der eingehenden Informationen, dem Erkennen oder Verstehen, folgt das Verarbeitungssystem bestimmten Wahrnehmungsprinzipien, die das Zusammensetzen der Informationen erleichtern. Dazu gehören:

- die Fähigkeit, die Welt in Gegenstände zu gliedern und zu strukturieren;

- die Fähigkeit, die nichtsprachlichen und später auch die sprachlichen Handlungen und Verhaltensweisen zeitlich zu gliedern und zu strukturieren;

- die Fähigkeit, diese beiden gegliederten Welten, also die gegenständliche und ereignishafte einerseits und die abstrakte andererseits, lautlich, gestisch und handlungsbestimmt in Beziehung zu bringen;

- die Fähigkeit, symbolische Beziehungen zu erkennen (zum Beispiel, ich` und ,du‘ als Symbole für Sprecher und Adressat zu identifizieren);

- die Fähigkeit, Sprache kreativ zu gebrauchen.

Wie oben bereits besprochen: Bedeutung ist immer subjektiv und entsteht zum einen durch Assimilation an das Bekannte, zum anderen wird durch bestehende Bedeutungsmuster vorab bestimmt, was wie wahrgenommen werden kann. Diese Bedeutungsmuster passen sich durch Akkommodation gleichzeitig an das Neue an.

Vorwissen und Kontext spielen also bei der Informationsverarbeitung eine ganz wesentliche Rolle, denn das, was wahrgenommen wird, wird instinktiv mit bekannten Mustern oder Elementen (Merkmalen) von Mustern verglichen. Diesen Prozess kann man in die Teilschritte Filtern, Einordnen und Zuordnen unterteilen. Beim Filtern wird wichtige und unwichtige Information getrennt, beim Einordnen werden Begriffe in vorhandene Kategorien eingeordnet, beim Zuordnen werden semantische Beziehungen zwischen Begriffen geknüpft. Bekanntes lässt sich daher auch schneller und besser erkennen. Fehlende Informationen lassen sich bei dieser Mustererkennung meist aus dem Kontext erschließen. Häufig genügen daher wenige 
Elemente eines Ganzen, um das Erkennen und Verstehen zu sichern. Der Wahrnehmungsapparat bemüht sich ständig um die Konstruktion von Sinn.

\section{$4 \quad$ Assoziationsverfahren als Datenbasis}

Die Konstruktionsprozesse bilden kognitive, das heißt lexikalisch-semantische und pragmatische Relationen ab, die über unterschiedliche Teilnetze aktiviert werden können. Aus der interkulturellen Metaphorisierungsforschung ist deutlich erkennbar, wie sehr sich Menschen dabei von ihren kulturspezifischen Welt-Erfahrungen leiten lassen. Wir haben mit der Assoziationstechnik, die in der Psychologie seit langem eingesetzt wird, operiert. Dabei geht es im Wesentlichen darum, in einer bestimmten Zeit, zu einem bestimmten Begriff spontan die wichtigsten Assoziationen zu nennen. In gewisser Weise ist das Verfahren die psychologische Ergänzung zu Kookurrenzen und den Modellen von Kilian (2001) und Hallsteinsdóttir (2001).

\begin{tabular}{|l|l|l|l|}
\hline Frieden & Anzahl & Reaktion & Anzahl \\
\hline Reaktion & 13 & amour (Liebe) & 6 \\
\hline Taube & 8 & guerre (Krieg) & 6 \\
\hline Krieg & 5 & monde (Welt) & 6 \\
\hline Hoffnung & 3 & colombe (Taube) & 5 \\
\hline Weiß & 3 & blanc (weiß) & 4 \\
\hline auf Erden & 2 & Noel (Weihnachten) & 3 \\
\hline Freiheit & 2 & tranquilité (Ruhe) & 3 \\
\hline $\begin{array}{l}\text { Freude, Frie- } \\
\text { de,Freude,Eierkuchen, Frie- } \\
\text { densengel, Kirche, leben, See- } \\
\text { lenruhe, Sehnsucht, selten, } \\
\text { schön, stiftend, Stille, Utopie, } \\
\text { verzeihen }\end{array}$ & sérénité (Heiterkeit) & terre (die Erde) & 2 \\
\hline $\begin{array}{l}\text { brauchen wir, Demonstration } \\
\text { beauté, bonheur, bonté, doux, Gandi, }\end{array}$ & $\begin{array}{l}\text { indien, intérieure, joie, Nations-Unies, } \\
\text { religion, romaine, souhait, un jour } \\
\text { peut-être, vie }\end{array}$ & 2 \\
\hline
\end{tabular}

Tabelle 1: Anzahl der Reaktionen auf den Begriff FRIEDEN bei den Deutschen und Frankokanadiern nach Roche/Roussy-Parent (2006: 242)

Die positive Empfindung des FRIEDENS wird von beiden Versuchsgruppen mit der hellen Farbe weiss/blanc assoziiert. Bei den frankokanadischen Versuchspersonen findet dabei eine vereinfachte Assoziation von WUT mit SCHWARZ und FRIEDEN mit WEISS statt. Bei beiden Gruppen erscheint zudem der metaphorische Begriff Taube/colombe als Symbol des FRIEDENS. Die frankokanadischen Versuchspersonen nennen darüber hinaus auch den metaphorischen Begriff Noël (Weihnachten). 


\begin{tabular}{|c|c|c|c|}
\hline \multicolumn{2}{|l|}{ Freiheit } & \multicolumn{2}{|l|}{ Liberté } \\
\hline Reaktion & Anzahl & Reaktion & Anzahl \\
\hline Gefängnis & 5 & expression (Meinungsäußerung)) & 5 \\
\hline Statue & 4 & voyage (Reise) & 3 \\
\hline Grenzenlos & 3 & air (Luft) & 2 \\
\hline Weite & 3 & bonheur (Glück) & 2 \\
\hline Frieden & 2 & cheval (Pferd) & 2 \\
\hline Luft & 2 & choix (Wahl) & 2 \\
\hline Münchener Freiheit & 2 & cinquante-cinq (55) & 2 \\
\hline Reisen & 2 & démocratie (Demokratie) & 2 \\
\hline schön & 2 & fleuve (Strom) & 2 \\
\hline Urlaub & 2 & fraternité (Brüderlichkeit) & 2 \\
\hline Wichtig & 2 & statue (Statue) & 2 \\
\hline $\begin{array}{l}\text { Berge, Betrug, Brüderlichkeit, } \\
\text { Fahne, Feigheit, fliegen, fran- } \\
\text { zösische Revolution, Gleich- } \\
\text { heit, Gut, Heimat, Knast, kost- } \\
\text { bar, Liebe, Natur, Unabhän- } \\
\text { gigkeit, Uneingeschränktheit, } \\
\text { Vogel, wegfahren, wertvoll, } \\
\text { Wind, Wunsch, Zeit }\end{array}$ & je 1 & $\begin{array}{l}\text { auto, cage, célibataire, conditionnelle, } \\
\text { congé, dans mon coeur, défendre, faire } \\
\text { ce que je veux, finalement, humani- } \\
\text { taire, indépendance, n'est pas une } \\
\text { marque de yaourt, oiseau paix, para- } \\
\text { noïaque, patrie, Québec, radio, région, } \\
\text { respiration, révolution, s'arrête où } \\
\text { commence celle de l'autre, servitude, } \\
\text { totale, ville }\end{array}$ & je 1 \\
\hline
\end{tabular}

Tabelle 2: Anzahl der Reaktionen auf den Begriff FREIHEIT bei den Deutschen und Frankokanadiern nach Roche/Roussy-Parent (2006: 244)

In einer weiterführenden Studie hat Ramdan (2013) die Liste auch für Vergleiche mit marrokanischen Sprechern verwendet und sie dabei auch um einige Begriffe erweitert.

\begin{tabular}{|c|c|c|c|c|c|}
\hline \multicolumn{2}{|l|}{ pouvoir } & \multicolumn{2}{|l|}{ Macht } & \multicolumn{2}{|l|}{ السلطة (sulta) } \\
\hline Reaktion & Anzahl & Reaktion & Anzahl & Reaktion & Anzahl \\
\hline argent (Geld) & 8 & Politik & 9 & الثرطة (Polizei) & 5 \\
\hline $\begin{array}{l}\text { président (Präsi- } \\
\text { dent) }\end{array}$ & 6 & Geld & 8 & ملك (König) & 4 \\
\hline $\begin{array}{l}\text { politique (Poli- } \\
\text { tik) }\end{array}$ & 4 & König & 5 & القمع $\begin{array}{r}\text { (Unterdrückung) } \\
\text { (2) }\end{array}$ & 3 \\
\hline puissance (Kraft) & 3 & Herrschaft & 4 & $\begin{array}{r}\text { (Regierung) } \\
\text { الحكومة }\end{array}$ & 3 \\
\hline $\begin{array}{l}\text { responsabilité } \\
\text { (Verantwortung) }\end{array}$ & 3 & Krieg & 2 & الاستبداد (Autorität) & 2 \\
\hline influence (Ein- & 2 & Gewalt & 2 & نفوذ (Herrschaft) & 2 \\
\hline
\end{tabular}


Akkommodationsprozessen

\begin{tabular}{|c|c|c|c|c|c|}
\hline fluss) & & & & & \\
\hline $\begin{array}{l}\text { règne } \\
\text { (Herrschaft) }\end{array}$ & 2 & Diktator & 2 & الدولة (Staat) & 2 \\
\hline savoir (wissen) & 2 & & & & \\
\hline devoir (Aufgabe) & 2 & & & & \\
\hline $\begin{array}{l}\text { Or, force, capita- } \\
\text { lisme, vouloir, } \\
\text { suprême, rébel- } \\
\text { lion, danger, or- } \\
\text { gueil, état, exécu- } \\
\text { tif, autorité, vice, } \\
\text { dictateur, diri- } \\
\text { geant, méchant, } \\
\text { entreprise, direc- } \\
\text { tion, trône, arabe, } \\
\text { pourquoi?, peur, } \\
\text { roi, petit }\end{array}$ & 1 & $\begin{array}{l}\text { Ungut, Star Wars, } \\
\text { Habitus, Wissen, } \\
\text { USA, Verantwor- } \\
\text { tung, Krone, } \\
\text { Kraft, grau, } \\
\text { Machthaber, Boss, } \\
\text { kalt, hungrig, aus- } \\
\text { nutzen, Ohnmacht, } \\
\text { regieren, Gier, } \\
\text { Missbrauch, groß, } \\
\text { machen, Konzerne, } \\
\text { gefährlich, Pharao }\end{array}$ & 1 & 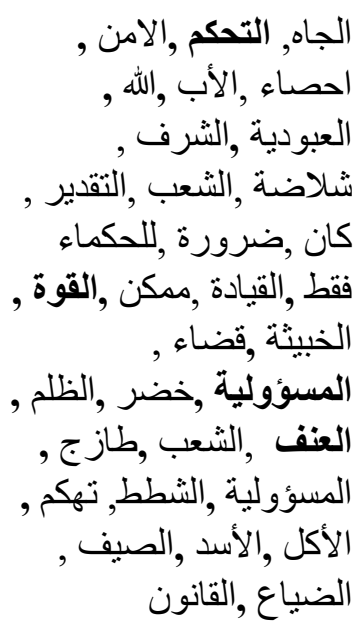 & 1 \\
\hline
\end{tabular}

Tabelle 3: Anzahl der Reaktionen auf den Begriff Macht bei den Deutschen, den Marokkanern und den Franzosen (cf. Ramdan (2013: 57)

Zu dem Begriffsfeld von ,Macht' etwa kommt er zu folgenden Ergebnissen. Dabei sind einige Gemeinsamkeit (zum Beispiel in Bezug auf die Merkmale ,König‘ und ,Herrschaft‘) bemerkenswert, aber ebenso die großen kulturspezifischen Unterschiede in den semantischen Feldern.

Diese exemplarischen Beobachtungen sind in zweifacher, konträr erscheinender Hinsicht für unser Thema interessant. Einerseits zeigen sich deutliche interkulturelle Bedeutungs- und Deutungsunterschiede. Das heißt, die Bedeutung ist klar von der spezifischen Wahrnehmung bestimmt. Im Durchschnitt der Bewertungen ergeben sich bei den Konkreta Medianwerte von 0,32 und bei den Abstrakta von nur 0,26, wobei ein Wert von 1,0 die komplette Übereinstimmung darstellen würde und wegen der Kulturspezifik der Metaphorisierung tatsächlich eine geringere Übereinstimmung der Abstrakta zu erwarten ist.

\begin{tabular}{|l|l|l|l|}
\hline & Adjektiv & $\begin{array}{l}\text { Kon- } \\
\text { kreta }\end{array}$ & Abstrakta \\
\hline Mittelwert & 0,300 & 0,319 & 0,273 \\
\hline Median & 0,25 & 0,32 & 0,26 \\
\hline
\end{tabular}

Tabelle 4: Die mittleren Übereinstimmungskoeffizienten der Adjektive, Konkreta und Abstrakta nach Roche/Roussy-Parent (2006: 237) 
Hieraus ergibt sich ein Bild der qualitativen Differenzen der Begriffsfelder und es eröffnen sich Einblicke in kulturspezifische Strategien der Begriffserschließung und Vernetzung. ${ }^{1}$ Die Übereinstimmungskoeffizienten sind erstaunlich niedrig, selbst bei eindeutigen Begriffen wie ,Mond '/,lune'.

$\begin{array}{ll}\text { Frieden - paix } & 0,45 \\ \text { Freiheit - liberté } & 0,24 \\ \text { Mond - lune } & 0,5 \\ \text { Sorge - trouble } & 0,1 \\ \text { Stolz - fierté } & 0,14\end{array}$

Konstruktionsprozesse, wie sie im Bereich der Semantik deutlich werden, bestimmen auch die Entwicklung der Grammatik. Demnach entsteht die Grammatik auf der Basis des Lexikons und damit letztlich auf der Basis von Bedeutungskonstruktion auf Grund von Welterfahrung. Die kulturspezifischen Wahrnehmungen und interkulturellen Differenzen sind also der eine Aspekt der Metaphorisierungen, die zu Stabilisierungen führen. Andererseits erklärt der Rückgriff auf körperliche Quellendomänen, trotz aller plausiblen kulturspezifischen Einflüsse, warum die Bedeutung der Begriffe in unterschiedlichen Sprachen dennoch so viele Gemeinsamkeiten aufweist (vergleiche hierzu die Arbeiten zur kognitiven Didaktik und zu Grammatikanimationen Roche/Suñer 2014; Suñer 2013).

Betrachten wir die Gemeinsamkeiten der Bedeutungen und Wahrnehmungen nun aber noch etwas genauer in Bezug auf gruppenspezifische Eigenschaften, stellen wir Folgendes fest: Lambert/Moore (1966), die als erste mit derartigen interkulturellen Vergleichen von eigentlich aus der Psychologie stammenden Assoziationsexperimenten gearbeitet haben, haben bemerkt, dass die Gruppe ihrer anglo-amerikanischen Versuchspersonen eine höhere Homogenität in den Assoziationen aufweist, als die Gruppe der französischen Teilnehmer, die also eine größere Heterogenität aufweist. Bilinguale franko-kanadische Versuchspersonen wurden später in dem Spektrum zwischen den beiden Gruppen angesiedelt. Das homogenere Verhalten bezeichnen Lambert und Moore als ,stereotypy` (Stereotypie).

In diesem Sinne verhalten sich unsere deutschsprachigen Informanten bei Adjektivbewertungen viel stereotypischer als unsere Franko-Kanadier. Während diese eine Reihe von Prototypen heranziehen, die ein Konzept bezeichnen (cf. Rosch/Mervis 1976), tendieren die Deutschen zur Verwendung von Antonymen. Ein Viertel aller Nennungen der deutschen Gruppe besteht aus Antonymen. Bei den Franko-Kanadiern liegen diese nur bei ,sucré',,mou', und ,dur' vor.

\footnotetext{
${ }^{1}$ Zur Notation: Um Missverständnisse zu vermeiden, werden Konzepte durchweg in Großbuchstaben geschrieben. Der Begriff STOLZ soll zum Beispiel nicht als das deutsche Wort Stolz beziehungsweise das französische Wort fierté, sondern als das Bezeichnete, das heißt der Begriff, verstanden werden. Wörter als Symbolzeichen werden kursiv geschrieben werden (siehe Dorn 1998). Bei etymologischen Erklärungen werden indogermanische Wörter auch kursiv geschrieben und die deutsche Entsprechung steht in einfachen Anführungszeichen. Hierbei weist das Symbol , *' darauf hin, dass das Wort nicht belegt, sondern rekonstruiert ist. Die fett gedruckten Reaktionen markieren Übereinstimmungen in beiden Sprachen.
} 
Akkommodationsprozessen

Nach Cramer (1968) kann die Messung der Varianz der innersprachlichen Reaktionen mittels eines distributiven Wertes erfolgen. Die Distribution $\alpha$ gibt das Verhältnis der Anzahl der genannten Reaktionswörter zu der Gesamtzahl der Nennungen an. Je größer der Wert ist, desto disparater sind die Antworten. In der Graphik sind die Distributionswerte für alle drei Kategorien von Begriffen der Wortliste zusammengestellt. Dabei ist ersichtlich, dass die abstrakten Begriffe in beiden Sprachen eine höhere Distribution besitzen als die konkreten Begriffe.

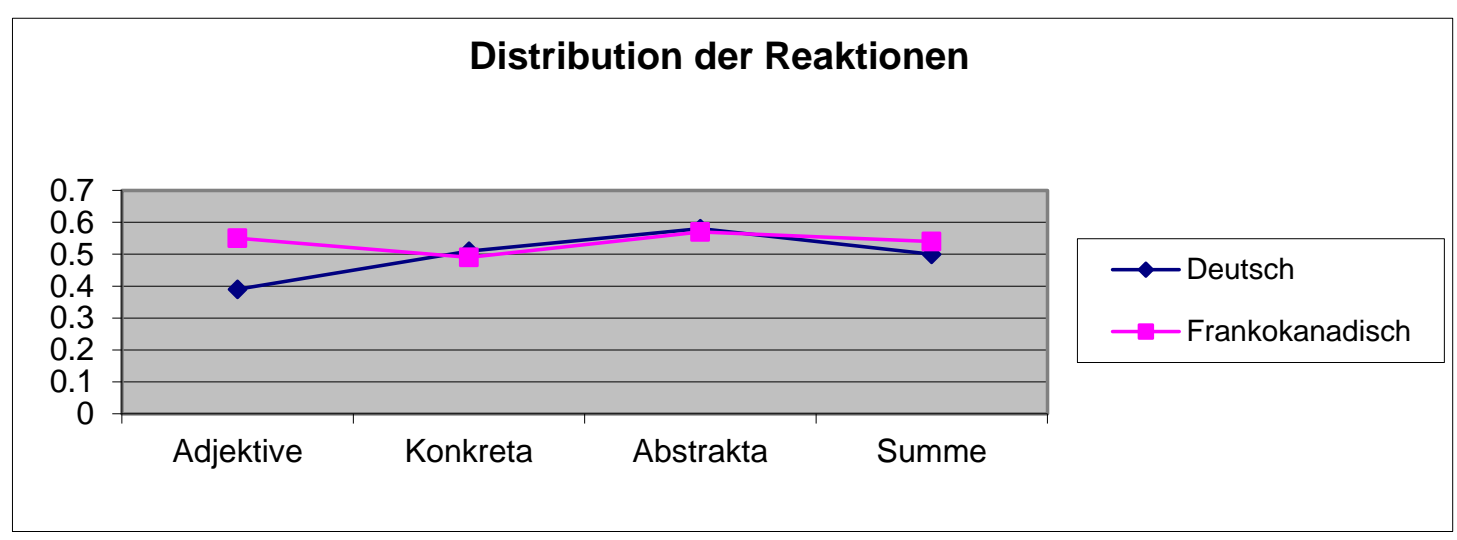

Graphik 1: Die mittleren Distributionskoeffizienten der Adjektive, Konkreta und Abstrakta im Deutschen und im Frankokanadischen nach (Roche/Roussy-Parent 2006: 245)

Was Lambert und Moore hier allerdings als Stereotypie bezeichnen, ist kaum auf vorgefertigte, die Wahrnehmung begrenzende oder gegebenenfalls negativ-zuschreibende Klassifizierungen zurückzuführen, sondern ergibt sich aus der „Natur der Sache“, aus den persönlichen, meist körperlichen Erfahrungen der Menschen, die solche Metaphern verwenden. Mit in diesem Sinne „stereotyp-begründeten“ Wahrnehmungsgewohnheiten wird ein Lerner in der Regel auch an eine fremde Kultur herangehen. Er wird also Konzepte übertragen und bei feststellbaren Differenzen den Transfer unterschiedlich bewerkstelligen (je nach Motivationsfaktoren, Sprachlerninteresse und -eignung etc.). Es ergibt sich daraus aber überhaupt nicht, warum eine Differenzierung dieser Stereotypie von Lernern prinzipiell nicht zu leisten sein soll, wenn sich die Erfahrungen entsprechend ändern. Warum soll ein Lerner nicht lernen können, dass der Bäckerei im Dänischen die Küche im Deutschen entsprechen kann: Wem es in der Küche zu heiß wird entspricht im Dänischen den der ikke kan lide lugten i bageriet (,der, der den Geruch in der Bäckerei nicht mag'); ruhig Blut bewahren kann mit ,kaltem Wasser im Blut' auch ausgedrückt werden (slå koldt vand $i$ blodet ,kaltes Wasser ins Blut schlagen'); oder Wasserrohren, dem grape vine oder der Gerüchteküche kann man gleichermaßen zuhören, auch wenn man sie vielleicht gar nicht kennt (lytte på vandrørene ,den Wasserrohren zuhören/Gerüchte über etwas hören').

\section{$5 \quad$ Zum Grad der Stereotypisierung}

Genau aus diesem Grund kann man auch davon ausgehen, dass andere, im traditionellen Verständnis „stereotype“ Vorerfahrungen und Einstellungen bei unterschiedlicher Erfahrung in Wirklichkeit veränderbar sind, sofern die andersartigen, neuen Erfahrungen den Wahrnehmungsapparat erreichen. Das geschieht nicht automatisch durch schlichte Begegnung mit Neuem. Wir wissen, dass deshalb etwa Schüler- und Studienaustauschprogramme, die auf schlichter Begegnung basieren, nicht zwangsläufig zu einer Reduzierung stereotyper Vorannahmen führen. So bleiben etwa sehr plakative Assoziierungen (eventuell: wie Bier oder 
Wurst zu Deutschland) trotz individueller Begegnungen hartnäckig bestehen, und positive Eigenschaften in Bezug auf die Bevölkerung des Gastlands erfahren teils weniger Zustimmung als in einer vergleichbaren Personengruppe (siehe die Studien von Sato-Prinz 2011, 2016). Oft bedarf es vielmehr der direkten Kontrastierung, der Provokation und der AhaErlebnisse, um die Auseinandersetzung, Reflexion und Modifikation in Gang zu setzen. Wie oben am Beispiel von Einstellungen zum Thema ,Gewalt und Widerstand' illustriert. Das Ergebnis ist nicht zwangsläufig das Vergessen oder Aufgeben etablierter und stabilisierter Einstellungen, sondern der Erwerb weiterer, differenzierterer Einstellungen auf Grund von Assimilations- und Akkommodationsprozessen, die neben anderen bestehen bleiben und sich gegebenenfalls weiter entwickeln. Der Unterschied zwischen stereotypisch-stabilisierten und „normalen“"Wahrnehmungsmustern ist also eher ein gradueller. Auf diese Weise können unterschiedliche Einstellungen rezeptiv und produktiv nebeneinander zur Verfügung stehen. Und wir benötigen sie. Wer etwa Komik und Satire verstehen will, kann dies nur, wenn er auch die karikierten Stereotype kennt. Im allgemeineren Sinne sind stabilisierte Wahrnehmungen normale Zwischenprodukte von Wahrnehmungsprozessen, die wir als Orientierungen und Grundlagen für die Entwicklung weiterer Perspektiven benötigen.

Halten wir als weiteres Ergebnis fest, dass die genannten Gemeinsamkeiten in der Metaphorik und der Metaphorisierungsdynamik in der Sprach- und Kulturvermittlung bisher fast völlig ungenutzt bleiben, weil Lehrkräfte und Verlage so viel Angst vor der vermeintlichen Elaboriertheit und Unsicherheit von Metaphern haben (cf. Roche 2013; Azuma/Littlemore 2010; Littlemore 2009; Danesi 2008; Littlemore/Low 2006). Um dieser didaktischen Ressourcenverschwendung Abhilfe zu schaffen, indem die Lernaufgabe des Lerners konstruktiv benannt wird, habe ich den Begriff der ,Transferdifferenz' (Roche/Suñer 2014; Roche 2013) in die Diskussion eingeführt. Die Bewältigung dieser Lernaufgabe, also die adäquate Übertragung von lingua-kulturellen Konzepten von einer Sprache auf die andere, resultiert folglich in einer konzeptuellen Kompetenz (,,conceptual fluency“ bei Danesi 2008).

Auflösen lassen wird sich das Fremde dabei nicht, nicht immer oder nicht vollständig. Die Skeptische Hermeneutik und die kognitive Psychologie verweisen vielmehr darauf, dass wir das Fremde als Katalysator für das Lernen brauchen. Es fordert die etablierten Wissens-, Bedeutungs- und Einstellungsbestände heraus und sorgt damit für die besagte Dynamik des Verstehens im Sinne von Transkulturationsprozessen. Hierzu gehören dynamische, transdifferente Einstellungen und Wahrnehmungen, die nebeneinander stehen und auch wieder zerfallen können, also eine unterschiedliche Halbwertzeit haben. Stereotype müssen nicht bestehen bleiben, zumindest wenn erwartet werden sollte, dass die Lerner sich auf Fremdes einlassen wollen, wie man das ja im Spracherwerb unterstellen sollte. Wo es tatsächlich zu Verfestigungen kommen könnte, lässt sich mit einer kontrastiven Didaktik die Dynamik wieder anschieben beziehungsweise in Gang halten.

\section{Literatur}

Azuma, Masumi/Littlemore, Jeannette (2010): "Promoting creativity in English language classrooms". JACET Kan-sai Journal 12: 8-19. 
Akkommodationsprozessen

Bennett, Milton J. (1993): "Towards ethorelativism. A developmental model of intercultural sensitivity”. In: Raigie, R. Michael (ed.): Education for the Intercultural Experience. Yarmouth ME, Intercultural Press: 21-71.

Cramer, Phebe (1968): Word Association. New York: Academic Press.

Danesi, Marcel (2008): “Conceptual errors in second-language learning”. In: de Knop, Sabine/de Rycker, Teun (eds.): Cognitive Approaches to Pedagogical Grammar. Berlin/New York, Mouton de Gruyter: 231-256.

Dorn, Matthias (1998): Priming-Effekte bei unterschiedlichen assoziierten Wortpaaren. Untersuchungen zum Status von Assoziationserhebungen und verschiedenen semantischen Relationen im Leseprozeß. Freiburg: IIG.

Hall, Edward (1959): The Silent Language. New York: Anchor Press.

Hall, Edward (1981): Beyond Culture. New York: Doubleday.

Hallsteinsdóttir, Erla (2001): Das Verstehen idiomatischer Phraseologismen in der Fremdsprache Deutsch. Hamburg: Kovač.

Hofstede, Geert (1991): Cultures and Organizations. UK: McGraw-Hill.

Ifenthaler, Dirk (2006): Diagnose lernabhängiger Veränderung mentaler Modelle. Entwicklung der SMD-Technologie als methodologisches Verfahren zur relationalen, strukturellen und semantischen Analyse individueller Modellkonstruktionen. Disseration: Universität Freiburg.

Kilian, Jörg (2001): „Kritische Semantik. Für eine wissenschaftliche Sprachkritik im Spannungsfeld von Sprachtheorie, Sprachnorm, Sprachpraxis“. Zeitschrift für germanistische Linguistik 29: 293-318.

Krusche, Dietrich/Krechel, Rüdiger (1984): Anspiel. Konkrete Poesie im Unterricht Deutsch als Fremdsprache. Bonn: Inter Nationes.

Lambert, Wallace E./Moore, Nancy (1966): "Word-association responses: comparisons of American and French monolinguals with Canadian monolinguals and bilinguals". Journal of Personality and Social Psychology 3: 313-320.

Littlemore, Jeannette (2009): Applying Cognitive Linguistics to Second Language Learning and Teaching. New York: Macmillan.

Littlemore, Jeannette/Low, Graham (2006): Figurative Thinking and Foreign Language Learning. New York: Macmillan.

Ortiz, Fernando (1995): Cuban Counterpoint. Tobacco and Sugar. Translated from Spanish by de Onis, Harriet. Durham: Duke University Press.

Ramdan Ait, Mohcine (2013): „Wortassoziationen: Ein interkultureller Vergleich zwischen dem Deutschen, dem Arabischen und dem Französischen“. Zeitschrift für Interkulturellen Fremdsprachenunterricht 18/1: 35-61.

Roche, Jörg (2013): Mehrsprachigkeitstheorie. Erwerb, Kognition, Transkulturation, Ökologie. Tübingen: Narr.

Roche, Jörg/Webber, Mark (1995): Für- und Widersprüche. Yale: Yale University Press.

Roche, Jörg/Roussy-Parent, Mélody (2006): „Zur Rolle der kontrastiven Semantik in interkultureller Kommunikation“. Fremdsprachen Lehren und Lernen 35: 228-250.

Roche, Jörg/Suñer Muñoz, Ferran (2014): „Kognition und Grammatik: Ein kognitionswissenschaftlicher Ansatz zur Grammatikvermittlung am Beispiel der Grammatikanimationen“. Zeitschrift für Interkulturellen Fremdsprachenunterricht 19/2: 119-145. 
Rosch, Eleanor/Mervis Carolyn/Johnson, David (1976): "Basic objects in natural categories". Cognitive Psychology 8: 382-439.

Sato-Prinz, Manuela (2011): „Zum Einfluss von Studienaustauscherfahrung auf das Deutschlandbild japanischer Studierender - Ergebnisse einer Querschnittstudie“. Zeitschrift für Interkulturellen Fremdsprachenunterricht 16/2: 185-203.

Sato-Prinz, Manuela (2016): Deutschlandbilder und Studienaustausch - Zur Veränderung von Nationenbildern im Rahmen von Studienaustauschaufenthalten am Beispiel japanischer Austauschstudierender in Deutschland. Unpublizirte Dissertation LMU München.

Scollon, Ron/Scollon, Suzanne (1990): “Athabaskan-English interethnic communication”. In: Carbaugh, Donal (ed.): Cultural Communication and Intercultural Contact. Hilsdale, Lawrence Erlbaum Associates: 259-286.

Suñer Muñoz, Feran (2013): „Bildhaftigkeit und Metaphorisierung in der Grammatikvermittlung am Beispiel der Passivkonstruktion“. Zeitschrift für Interkulturellen Fremdsprachenunterricht 18/1: 4-20.

Thomas, Alexander (2005): Grundlagen der interkulturellen Psychologie. Nordhausen: Bautz.

Trompenaars, Fons (1993): Riding the Waves of Culture. London: The Economist Books.

Welsch, Wolfgang (1999): "Transculturality - the Puzzling Form of Cultures Today". In Featherstone, Mike/Scott, Lash (eds.): Spaces of Culture: City, Nation, World. London, Sage Publications: 194-213.

Welsch, Wolfgang (2010): „Was ist eigentlich Transkulturalität?“ In: Darowska, Lucyna et al. (eds.): Hochschule als transkultureller Raum? Kultur, Bildung und Differenz in der Universität. Bielefeld, Transcript: 39-66. 